\section{Jun-Hwan Kim, Young-Joon Kwon}

Department of Neurosurgery, Kangbuk Samsung Hospital, Sungkyunkwan University School of Medicine, Seoul, Korea

\section{Corresponding Author:}

Young-Joon Kwon

Department of Neurosurgery,

Kangbuk Samsung Hospital,

Sungkyunkwan University School of Medicine, 29 Saemunan-ro, Jongno-gu, Seoul 03181, Korea

Tel: +82-2-2001-2450

Fax: +82-2-2001-2157

E-mail: neuriac@gmail.com

Received: July 12, 2017

Revised: August 30, 2017

Accepted: August 31, 2017

\title{
Long-term Clinical and Radiological Outcomes after Central Decompressive Laminoplasty for Lumbar Spinal Stenosis
}

Objective: There are many technical modifications of decompressive lumbar laminectomy. The purpose of this study was to report long-term clinical and radiological outcomes of central decompressive laminoplasty (CDL), the corresponding author's own modification of lumbar laminectomy for lumbar spinal stenosis (LSS).

Methods: Among 100 patients who underwent CDL by a single surgeon between December 2010 and March 2014, 68 patients were included in this study. Mean follow-up time was 37.7 months. Clinical and radiological data were gathered prospectively and reviewed retrospectively. Clinical outcome was measured by using visual analog scale (VAS) for back/buttock and leg, and the Oswestry Disability Index (ODI). Radiological outcome was measured by neutral slippage percentage, dynamic slippage percentage, and dynamic intervertebral angel on sagittal X-ray. Outcomes after CDL were assessed by changes of clinical and radiological parameters from the baseline. Mixed effect model with random patients' effect as used to test for differences in the repeated measured clinical and radiological data.

Results: The patients had no serious complications with an uneventful recovery during the early postoperative period. In the early postoperative period, VAS scores for back/buttock and leg improved significantly and were kept with time $(p<0.001)$. ODI also improved significantly during the postoperative follow-up period $(p<0.001)$. The radiologic parameters were well maintained and showed no progression of instability. During the follow-up, a case of herniated disc at same level recurrence was noted after lifting trauma, and 2 adjacent foraminal stenosis needed additional surgery.

Conclusion: $\mathrm{CDL}$ provides long-term pain relief and functional restoration without progression of radiological instability.

Key Words: Lumbar spine, Laminectomy, Surgical procedures, Stenosis, Patient outcome assessment

\section{INTRODUCTION}

Lumbar spinal stenosis (LSS) is a well-known cause of low back pain and neurogenic claudication which cause disability, especially in aged people. Nonsurgical conservative treatments including activity modification (core muscle strengthening exercise, posture change, and restriction of activity), medication, epidural steroid injections are helpful for patients with mild to moderate symptoms. If conservative treatments fail, surgical intervention will be considered as the next treatment. Surgical decompression for LSS is indicated in a patient with intractable pain, neurogenic claudication, and motor weakness, accompanied by compromised spinal canal.

Conventional laminectomy is the most common surgical approach for decompression of spinal stenosis, but it may cause fibro-muscular and bony structure damage leading to significant instability, which results in poor long-term prognosis. And extensive resection of the structures can be associated with extensive perioperative blood loss, prolongation of postoperative pain, and the delay of hospital stay ${ }^{19)}$. There are many technical modifications for decompression to relieve the problem associated with the conventional laminectomy ${ }^{3,5,8,21,26}$.

Central decompressive laminoplasty (CDL) was introduced as one of the modifications of the decompressive surgery. In the previous stu$\mathrm{dy}$, the author reported the surgical techniques and early surgical outcome ${ }^{15}$. The aim of this study was to present the long-term clinical and radiological outcomes of CDL for the treatment of LSS. 


\section{MATERIALS AND METHODS}

Between December 2010 and March 2014, 100 patients with LSS underwent CDL by a single surgeon (YJK). The patients underwent consistent baseline clinical and radiological investigation, and adequate follow-up over 12 months was possible in 68 patients. Most patients have low back/buttock and leg pain, and/or claudication. All patients underwent adequate conservative treatment including pain medicine, physical therapy, and nerve block for over 6 weeks before the operation. LSS was identified on magnetic resonance imaging and/or computed tomography (CT) with 3-dimensional reconstruction. Preoperative dynamic X-rays were taken of all patients to diagnose significant spinal instability. LSS accompanied with herniated disc, or grade 1 degenerative spondylolisthesis was also included. Patients who had a high grade (over grade 2) spondylolisthesis and stenosis accompanied with the traumatic or neoplastic disease were excluded from this analysis.

The Institutional Review Board of Kangbuk Samsung Hospital approved the study protocol (KBSMC 201707026). Informed consent was waived by the board.

\section{Outcome Assessment}

The pain was measured separately for back/buttock and leg according to a patient-reported outcome with a 10-point visual analog scale (VAS). Functional disability was checked using the Oswestry Disability Index (ODI), which has been culturally validated for Korean-language speakers ${ }^{10)}$.

Radiological parameters are assessed by lateral X-ray on neutral position and dynamic posture with flexion and extension. The parameters were evaluated to determine the progression of spinal instability. Progression of spondylolisthesis was assessed on neutral and dynamic position. It was calculated by neutral slip percentage, the slippage divided by the length of the upper line at the lower vertebra, and dynamic slip percentage, the difference in the slip percentage between the flexion and extension posture. The dynamic intervertebral angle was calculated by the difference in intervertebral angles during flexion and extension (Fig. 1). Radiographs were analyzed using the PiView STAR pro- gram (INFINITT Healthcare, Seoul, Korea).

Outcomes after CDL were analyzed using changes of clinical and radiological parameters from the preoperative baseline. Postoperatively, the parameters were surveyed at 3 months, 6 months, 1 year, 2 years, 3 years, and thereafter. For the patients following more than 3 years, the last follow-up data were selected for analysis. An independent researcher conducted the survey at the time of follow-up. Data was collected prospective manner and analyzed retrospectively via electronic medical record and image software review.

\section{Statistical Analysis}

The mixed-effect model was used to test for differences from baseline in VAS scores of back/buttock, VAS scores of leg, and ODI measured repeatedly within a patient. Random effects were each parameter for patients, and error. Fixed effects were times at baseline and postoperative periods. Multiple comparisons between baseline and each follow-up time value were adjusted by Bonferroni method. Data were analyzed using the IBM SPSS Statistics ver. 24.0 (IBM Co., Armonk, NY, USA). A p-value of $<0.05$ was considered statistically significant.

\section{Surgical Technique}

Detailed surgical technique and related images were described in the previous study ${ }^{15}$. Brief technique is as follows. Under the general anesthesia, the patient was positioned on the Wilson frame, and surgical level checked using a C-arm fluoroscopy. After midline 3-cm-sized skin incision is performed over the spinous process and down to supraspinous and interspinous ligament. The sharp incision onto the ligaments enabled the functional soft tissue closure after primary operation. During bilateral dissection of the muscles exposing laminae of vertebrae, special care was done not to violate the facet capsule. After retraction of dissected muscle bilaterally, a small portion of the inferior spinous process of the cranial vertebra is removed, and the superior portion of the lamina in caudal vertebra is removed with a bone rongeur. The spinolaminar junction of the vertebrae is drilled with a cutting burr. The prone positioning with elevated curvature of the frame and release of ligamentum flavum from
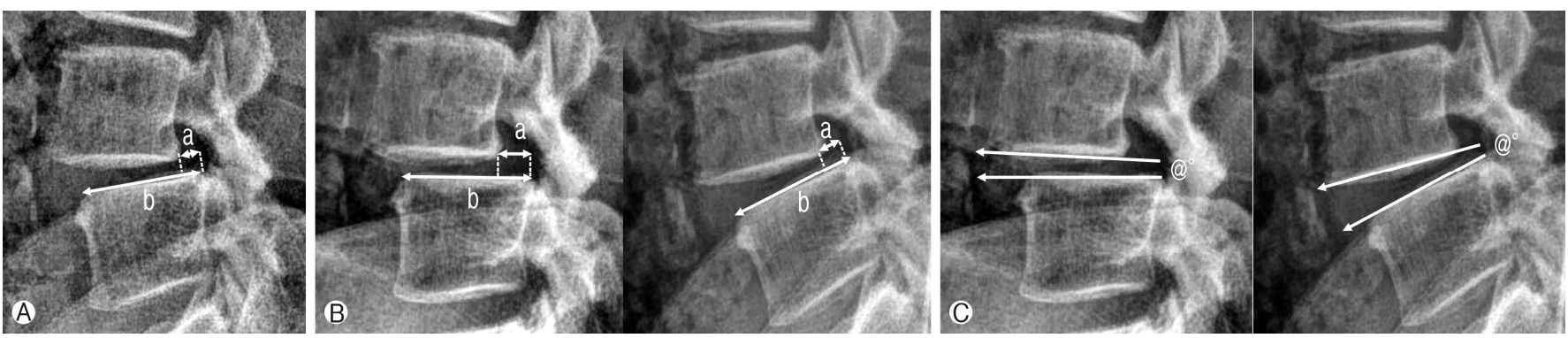

Fig. 1. (A) Neutral slip percentage: the slippage (a)/the length of the upper line of the lower vertebra (b). (B) Dynamic slip percentage: the difference in the slip percentage between flexion and extension posture. (C) Dynamic intervertebral angle (IVA): the difference in IVA during flexion and extension. 
the lamina opens interlamina waking space. The ligament was removed with a rongeur. The capsular portion of the ligament flavum was not disturbed to maintain facet joint stability. Using a high-speed drill and spinal punches, undercutting of caudal lamina was performed.

Decompression of nerve roots was performed from the contralateral side using an operating microscope. Tilting down the operative table to opposite side provide the surgical corridor to remove upper proximal exiting root. This contralateral approach made possible the undercutting of the facet joint while decompressing distal traversing root. The facet was removed in a tapered fashion, which allowed complete decompression of the root and sparing of the facet capsule (Fig. 2). Complete decompression of the root was performed along the pedicle to the lowermost part of the recess, at the turning point of the root

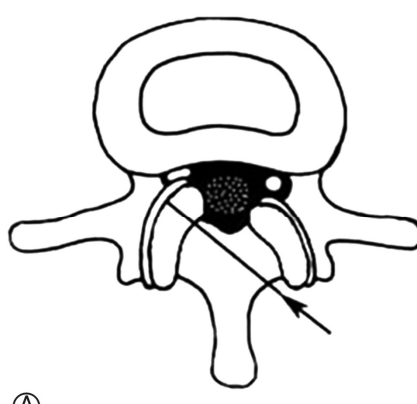

(A)

Fig. 2. Compressed root by hypertrophied articular processes (A) can be decompressed by inclined undercutting without disruption of facet joint and overlying facet capsule (B).

Table 1. Dermographic and clinical characteristics

\begin{tabular}{lc}
\hline \hline Characteristic & Value \\
\hline Age (yr), mean (range) & $61.6(31-81)$ \\
Sex, male:female & $30: 38$ \\
Symptom, n & \\
Low back pain & 48 \\
Radiating pain & 66 \\
Neurogenic intermittent claudication & 32 \\
Weakness (below IV-) & 19 \\
Stenotic level (discectomy level) & \\
L1/2 & $2(0)$ \\
L2/3 & $8(1)$ \\
L3/4 & $15(6)$ \\
L4/5 & $51(12)$ \\
L5/S1 & $10(6)$ \\
No. of levels underwent surgery, n (\%) & \\
1 & $50(73.5)$ \\
2 & $18(26.5)$ \\
Discectomy, n (\%) & $23(33.8)$ \\
Previous surgery, n (\%) & $9(13.2)$ \\
Additional surgery, n (\%) & $3(4.4)$ \\
Follow-up (mo), mean (range) & $37.7(12-85)$ \\
\hline
\end{tabular}

into the neural foramen. Discectomy was performed when soft herniated disc existed. The divided ligaments and skin were closed after an insertion of a drain.

\section{RESULTS}

Sixty-eight patients had adequate long-term clinical and radiological follow-up. Mean follow-up time was 37.7 months (range, 12-85 months). Average age at the time of surgery was 61.6 years (range, 31-81 years). Male to female ratio was 0.79 (male, 30; female, 38). CDL was done for 86 stenotic levels and L4/5 is the most frequent surgical level (51 levels, 59.3\%). Discectomy was done for 23 patients (33.8\%) and 25 levels (29.1\%). Fifty of 68 patients $(73.5 \%)$ were performed one-level surgery. Nine patients (13.2\%) underwent previous lumbar spine surgery from other surgeons (Table 1).

No serious complications were noted during perioperative and early postoperative period. There were 3 additional surgeries after initial CDL during the follow-up. A 64-year-old woman who underwent $\mathrm{CDL}$ on $\mathrm{L} 1 / 2$ and $\mathrm{L} 2 / 3$, one and half years later, required discectomy on the right side of $\mathrm{L} 1 / 2$ after lifting trauma. The other 2 patients had received upper and lower adjacent root decompression for far-lateral foraminal stenosis after 3 years and 1 year/3 months after the initial surgery, respectively.

Mean preoperative VAS scores of back/buttock (backVAS), VAS scores of leg (legVAS), and ODI were 4.3 (median, 5.0; interquartile range [IQR], 2.8-7.0), 5.6 (median, 5.5; IQR, 4.5-7.1), and 45.0 (median, 44.4; IQR, 34.5-55.6), respectively. The 3month follow-up data (backVAS; 1.7 [median, 1.0; IQR, 0-3.0], legVAS; 0.8 [median, 0; IQR, 0-1.1], ODI; 21.3 [median, 17.8; IQR, 8.9-30.0]) showed significant improvements vs. the baseline values $(p<0.001$, respectively). The mixed-effect model can analyze long-term trend of outcome after the surgery. It showed that back and leg VAS scores improved significantly in the early postoperative period ( 3 months) and maintained at plateau with time. ODI was lowest at 6 months postoperatively and tended to maintain plateau over the follow-up period ( $\mathrm{p}<0.001$, respectively) (Fig. 3).

Baseline mean neutral slippage percentage was 4.9 (median, 3.8; IQR, 0-8.5), and the baseline preoperative dynamic slippage percentage (the difference in the slip percentage during flexion and extension posture) was 3.5 (median, 1.8; IQR, 0.5-5.4). Another tool for assessing dynamic instability, dynamic intervertebral angle, was checked mean $7.6^{\circ}$ (median, 7.3 ; IQR, 4.110.1) at baseline. During the post-operative follow-up, the operative levels were well maintained without significant progression of spondylolisthesis in neutral position $(\mathrm{p}=0.186)$. Dynamic slippage and dynamic intervertebral angle were not increased over the follow-up time ( $p=0.236$ and $p=0.123$, respectively), which means good maintenance of spinal stability (Fig. 4).

\section{DISCUSSION}

Decompressive laminectomy has been known as a standard technique for treatment of degenerative LSS. Generally, the long- 

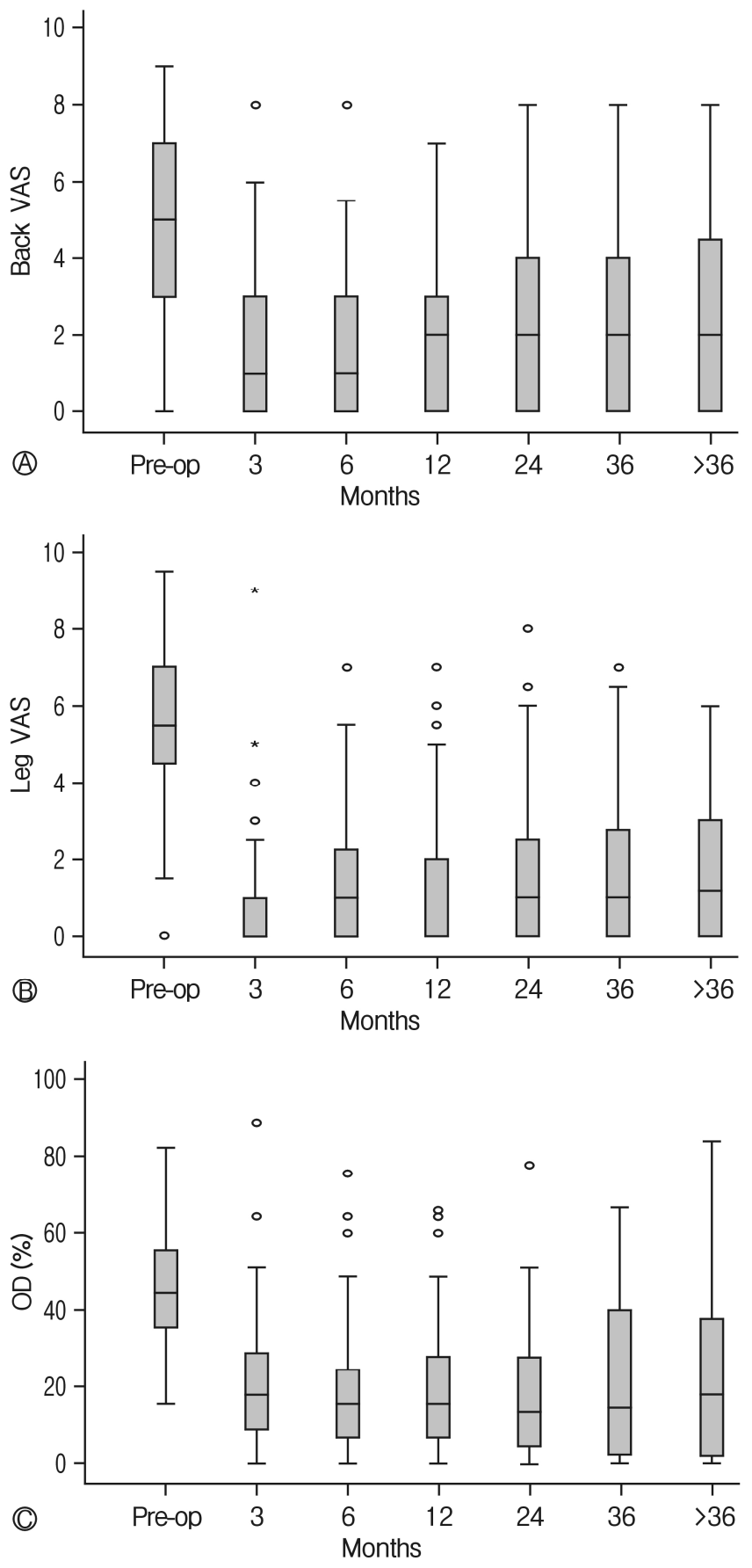

Fig. 3. Clinical outcomes measured by visual analog scale (VAS) and Oswestry Disability Index (ODI). VAS scores of back/buttock (backVAS) (A) and leg (legVAS) (B) decrease immediately in the early postoperative period. backVAS and legVAS slightly increase in 1 year and 2 years after central decompressive laminoplasty, respectively, and maintain at plateau with time. (C) ODI decrease immediately and maintain during the follow-up period. The bottom and top of the box represent the first and third quartile and the line inside the box is the median. The circles mean outliers beyond 1.5 interquartile ranges and the asterisk mean outliers beyond 3.0 interquartile ranges.
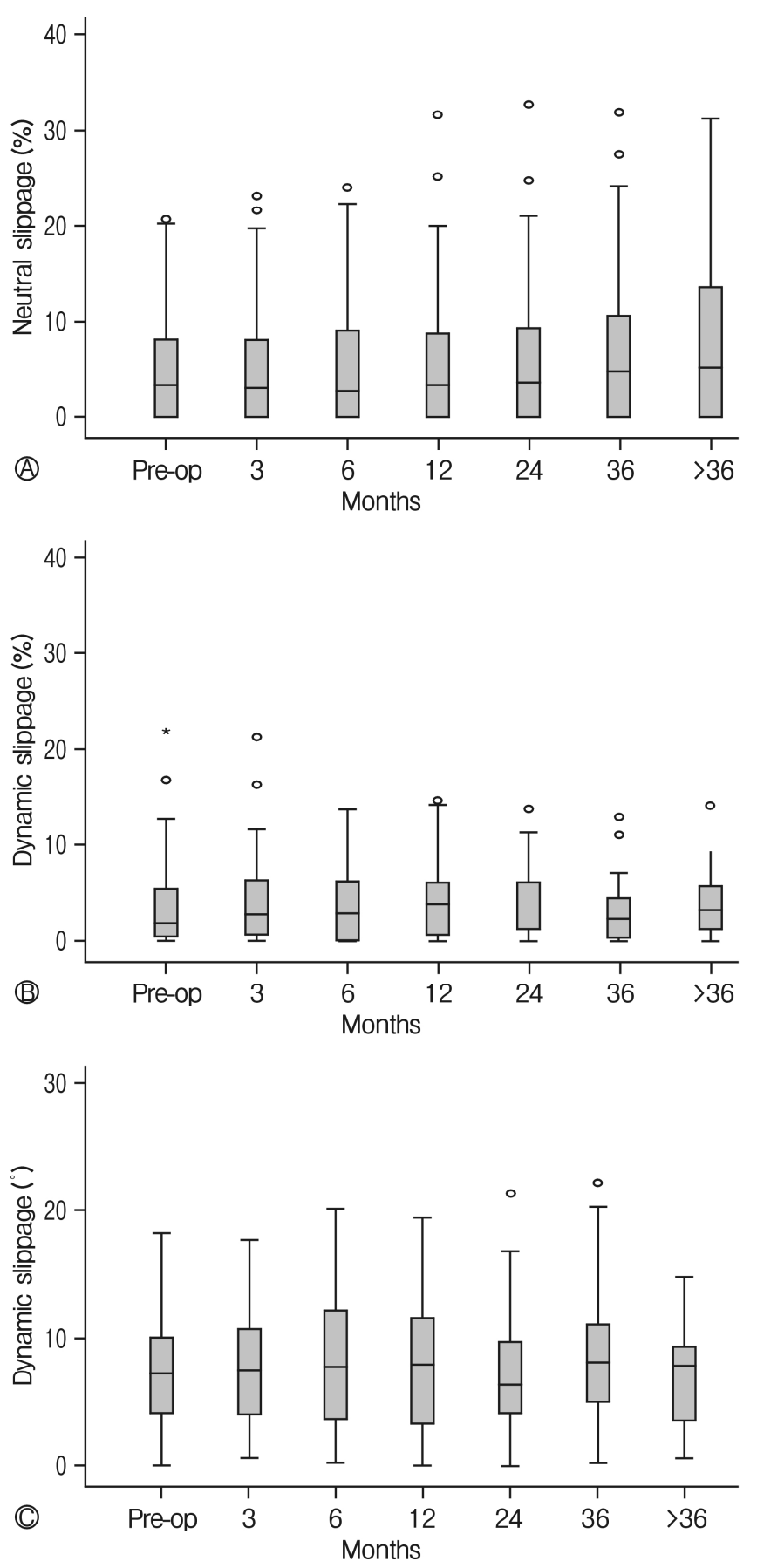

Fig. 4. Radiological outcomes were measured by neutral slip percentage (A), dynamic slip percentage (B), and dynamic intervertebral angle (C). All radiological outcomes are well maintained without significant progression of spondylolisthesis during the follow-up. The circles mean outliers beyond 1.5 interquartile ranges and the asterisk mean outliers beyond 3.0 interquartile ranges.

term prognosis of the technique has been reported poor as time passed by, despite wide variational and little data from long-term follow-up ${ }^{12,13,24)}$. Some studies reported good long-term results 
using minimal resection of the facet joint during sufficient neural decompression $^{5,22)}$, suggesting preservation of biomechanical stability, especially in the facet joint area, is required for long-term success of this technique.

Conventional total laminectomy could provide wide neural canal decompression but leads to extensive resection of supraspinous and interspinous ligaments, spinous process, and lamina. And it needs wide removal of facet joints that provide spinal stability. Compromise of facet joints may transmit direct compressive forces to the disc and longitudinal ligament, and cause degenerative spinal instability 7,18 .

Several technical modifications of conventional laminectomy have been suggested to overcome this limitation. Bilateral laminotomy ${ }^{3,8)}$ and unilateral laminotomy with bilateral decompression $^{4,11,20,25)}$ are well-known techniques. These methods enable surgeons to preserve midline structure while decompressing thecal sac and nerve roots, and showed good surgical outcomes ${ }^{4,9)}$. However, those have some technical shortcomings. First, preservation of midline supporting structure may interrupt the access to the ipsilateral lateral recess and foraminal area, especially if hypertrophied spinous existed. Next, large amount of facet resection is needed to access to the ipsilateral lateral recess and neural foramen, and violation of facet joint capsule occurs frequently. These may have an unfavorable effect on stability during the long-term follow-up. Recently, endoscopic surgery is another option of decompression for minimizing operation-related trauma. Although some authors published similar surgical outcome compared to previous microsurgical decompressive laminecto$\mathrm{my}^{14,17}$, the surgery is technically demanding in severe cases and has not enough data for safety and effectiveness ${ }^{16}$.

To overcome this limitation, we devised a specific modification of decompressive laminectomy, the CDL, which provide wide decompression while preserving facet stability by adequate bony resection limited in physiologic range ${ }^{15)}$. Using the surgical corridor into the central interspinous area, the technique preserves near-total facet joint including the capsule overlying the joint. Excellent surgical view and unhindered route to the central canal, lateral recess, and upper/lower foraminal area provide thorough decompression of the neural elements. CDL provided an excellent early surgical outcome with significant pain and functional improvement.

In the present study, the author displayed long-term results of CDL for LSS using both clinical and radiological parameters. The mixed-effect model was used to adjust for missing data for the repeatedly checked data. The statistical model was useful in the retrospective analysis of this prospectively collected data with long-term follow-up. VAS scores for back/buttock and leg improved significantly from baseline in the immediate postoperative period ( 3 months) and maintained at plateau with time $(\mathrm{p}<0.001)$. ODI improved significantly immediate postoperative period and maintained during the follow-up $(\mathrm{p}<0.001)$ without worsening of the symptom. These results were comparable to other long-term clinical outcomes of standard modification of decompressive laminectomy, unilateral laminotomy with bilateral decompression ${ }^{4,9)}$.
A systematic review for postoperative spondylolisthesis revealed that instability was seen in $12 \%$ of open laminectomy, and more frequently in pre-existing spondylolisthesis $(12.6 \%)^{6}$. CDL provided stable radiological outcome showing no progression of instability during this long-term follow-up study. Three representative parameters for progression of spondylolisthesis; neutral slippage, dynamic slippage, and dynamic intervertebral angle ${ }^{23)}$, were well maintained and not increased over the follow-up period. The facet joint and its capsule are key structures for spinal stability, which resist shear force $(33 \%)^{1)}$ and most of bending force $(70 \%)^{2)}$ in flexion position. As mentioned above, CDL preserves facet joint and capsule via inclined undercutting through the contralateral surgical trajectory. This could contribute to no progression of spondylolisthesis despite simple decompression without fusion.

This observational study of a long-term follow-up period, involved a relatively small number of patients. It is necessary to execute prospective controlled trials in a larger population with other laminectomy techniques (unilateral laminotomy with bilateral decompression, endoscopic laminotomy) or fusion surgery for confirmation of the current results.

\section{CONCLUSION}

CDL is a good surgical option for treating degenerative LSS. It can be easily applied, and allows excellent field visualization and decompression, sparing of ligament and bony structure to maintain the stability. It results in long-lasting pain relief and improved quality of life without progression of radiological instability.

\section{CONFLICT OF INTEREST}

No potential conflict of interest relevant to this article was reported.

\section{REFERENCES}

1. Adams MA, Hutton WC: The mechanical function of the lumbar apophyseal joints. Spine (Phila Pa 1976) 8:327-330, 1983

2. Adams MA, Hutton WC, Stott JR: The resistance to flexion of the lumbar intervertebral joint. Spine (Phila Pa 1976) 5:245-253, 1980

3. Aryanpur J, Ducker T: Multilevel lumbar laminotomies: an alternative to laminectomy in the treatment of lumbar stenosis. Neurosurgery 26:429-432, 1990

4. Cavuşoğlu H, Kaya RA, Türkmenoglu ON, Tuncer C, Colak I, Aydin Y: Midterm outcome after unilateral approach for bilateral decompression of lumbar spinal stenosis: 5-year prospective study. Eur Spine J 16:2133-2142, 2007

5. Getty CJ, Johnson JR, Kirwan EO, Sullivan MF: Partial undercutting facetectomy for bony entrapment of the lumbar nerve root. J Bone Joint Surg Br 63-B:330-335, 1981

6. Guha D, Heary RF, Shamji MF: Iatrogenic spondylolisthesis following laminectomy for degenerative lumbar stenosis: systematic 
review and current concepts. Neurosurg Focus 39:E9, 2015

7. Haher TR, O'Brien M, Dryer JW, Nucci R, Zipnick R, Leone DJ: The role of the lumbar facet joints in spinal stability. Identification of alternative paths of loading. Spine (Phila Pa 1976) 19:26672670, 1994

8. Herron LD, Pheasant HC: Bilateral laminotomy and discectomy for segmental lumbar disc disease. Decompression with stability. Spine (Phila Pa 1976) 8:86-97, 1983

9. Jang JW, Park JH, Hyun SJ, Rhim SC: Clinical outcomes and radiologic changes after microsurgical bilateral decompression by a unilateral approach in patients with lumbar spinal stenosis and grade I degenerative spondylolisthesis with a minimum 3-year follow-up. Clin Spine Surg 29:268-271, 2016

10. Jeon CH, Kim DJ, Kim SK, Kim DJ, Lee HM, Park HJ: Validation in the cross-cultural adaptation of the Korean version of the Oswestry Disability Index. J Korean Med Sci 21:1092-1097, 2006

11. Ji YC, Kim YB, Hwang SN, Park SW, Kwon JT, Min BK: Efficacy of unilateral laminectomy for bilateral decompression in elderly lumbar spinal stenosis. J Korean Neurosurg Soc 37:410-415, 2005

12. Jönsson B, Annertz M, Sjöberg C, Strömqvist B: A prospective and consecutive study of surgically treated lumbar spinal stenosis. Part II: Five-year follow-up by an independent observer. Spine (Phila Pa 1976) 22:2938-2944, 1997

13. Katz JN, Lipson SJ, Larson MG, McInnes JM, Fossel AH, Liang $\mathrm{MH}$ : The outcome of decompressive laminectomy for degenerative lumbar stenosis. J Bone Joint Surg Am 73:809-816, 1991

14. Komp M, Hahn P, Oezdemir S, Giannakopoulos A, Heikenfeld R, Kasch R, et al: Bilateral spinal decompression of lumbar central stenosis with the full-endoscopic interlaminar versus microsurgical laminotomy technique: a prospective, randomized, controlled study. Pain Physician 18:61-70, 2015

15. Kwon YJ: Central decompressive laminoplasty for treatment of lumbar spinal stenosis : technique and early surgical results. J Korean Neurosurg Soc 56:206-210, 2014

16. Lurie J, Tomkins-Lane C: Management of lumbar spinal stenosis. BMJ 352:h6234, 2016
17. Minamide A, Yoshida M, Yamada H, Nakagawa Y, Hashizume $\mathrm{H}$, Iwasaki $\mathrm{H}$, et al: Clinical outcomes after microendoscopic laminotomy for lumbar spinal stenosis: a 5-year follow-up study. Eur Spine J 24:396-403, 2015

18. Nakai O, Ookawa A, Yamaura I: Long-term roentgenographic and functional changes in patients who were treated with wide fenestration for central lumbar stenosis. J Bone Joint Surg Am 73:11841191, 1991

19. Overdevest G, Vleggeert-Lankamp C, Jacobs W, Thomé C, Gunzburg R, Peul W: Effectiveness of posterior decompression techniques compared with conventional laminectomy for lumbar stenosis. Eur Spine J 24:2244-2263, 2015

20. Park WB, Hong JT, Lee SW, Sung JH, Yang SH, Kim IS: Clinical and radiological comparison between ipsilateral and contralateral side canal decompression using an unilateral laminotomy approach. Korean J Spine 13:41-46, 2016

21. Poletti CE: Central lumbar stenosis caused by ligamentum flavum: unilateral laminotomy for bilateral ligamentectomy: preliminary report of two cases. Neurosurgery 37:343-347, 1995

22. Sanderson PL, Getty CJ: Long-term results of partial undercutting facetectomy for lumbar lateral recess stenosis. Spine (Phila Pa 1976) 21:1352-1356, 1996

23. Sasai K, Umeda M, Maruyama T, Wakabayashi E, Iida H: Microsurgical bilateral decompression via a unilateral approach for lumbar spinal canal stenosis including degenerative spondylolisthesis. J Neurosurg Spine 9:554-559, 2008

24. Scholz M, Firsching R, Lanksch WR: Long-term follow up in lumbar spinal stenosis. Spinal Cord 36:200-204, 1998

25. Spetzger U, Bertalanffy H, Naujokat C, von Keyserlingk DG, Gilsbach JM: Unilateral laminotomy for bilateral decompression of lumbar spinal stenosis. Part I: Anatomical and surgical considerations. Acta Neurochir (Wien) 139:392-396, 1997

26. Watanabe K, Hosoya T, Shiraishi T, Matsumoto M, Chiba K, Toyama Y: Lumbar spinous process-splitting laminectomy for lumbar canal stenosis. Technical note. J Neurosurg Spine 3:405-408, 2005 